\title{
Cutaneous Mucormycosis of the Interscapular Region in an Immunocompetent Patient
}

\author{
Authors: \\ *Pankil Doshi,, Yash Sanghvi,, Jwal Doctor,, Vaibhavi Parsaniya' \\ 1. Anand Surgical Hospital, Ahmedabad, Gujarat, India \\ 2. Institute of Pharmacy, Nirma University, Ahmedabad, Gujarat, India \\ *Correspondence to drpankilpdoshi@gmail.com \\ Disclosure: $\quad$ The authors have declared no conflicts of interest. \\ Received: \\ 09.10 .20 \\ Accepted: \\ 14.01.21 \\ Keywords: \\ Cutaneous mucormycosis, immunocompetent, interscapular region. \\ Citation: \\ EMJ Microbiol Infect Dis. 2021;2[1]:85-89.
}

\begin{abstract}
Mucormycosis is an invasive fungal infection caused by opportunistic fungi of the phylum Glomeromycotan, subphylum Mucormycotina, mainly affecting individuals with immunosuppression. Cutaneous mucormycosis is the third most common clinical form of the disease preceded by pulmonary and rhinocerebral mucormycosis. The usual factors predisposing to this infection are individuals who are immunocompromised with conditions like HIV, haematological malignancies, and diabetes mellitus, but a significant proportion of patients are immunocompetent. The agents of mucormycosis are abundantly present in nature and are transmitted to the skin by direct inoculation. It may be due to needle sticks, stings, and bites by animals, motor-vehicle accidents, natural disasters, and burn injuries. The clinical presentation is non-specific, but an indurated plaque that rapidly evolves to necrosis (eschar) is a common finding. The infection can invade locally, and also penetrate into the adjacent fat, muscle, fascia, and bone, or become disseminated. It is difficult to diagnose because of the non-specific presentation of mucormycosis. Biopsy and culture should be performed. Treatment consists of multidisciplinary management, including surgical debridement, use of antifungal drugs (amphotericin B and posaconazole), and reversal of underlying risk factors, when possible. Mortality rates are significant, ranging from $4 \%$ to $10 \%$ in localised mucormycosis infection, but are lower than the other forms of the disease. The authors present a case here of a 38-year-old immunocompetent male with cutaneous mucormycosis at the interscapular region.
\end{abstract}

\section{INTRODUCTION}

Mucormycosis is an uncommon but emerging opportunistic fungal infection with high morbidity and mortality, feared by clinicians worldwide. ${ }^{1}$ It is a potentially lethal infection caused primarily by filamentous fungus Rhizopus, Mucor, and Lichtheimia species of the fungi of the order Mucorales. ${ }^{2-6}$
It usually affects patients with poorly controlled diabetes and individuals with immunosuppression. ${ }^{7-11}$ Frequent clinical presentations include rhino-cerebral, pulmonary, and cutaneous forms, and less frequently, gastrointestinal, disseminated, and miscellaneous forms. ${ }^{12-16}$

This case report will focus on cutaneous mucormycosis. 


\section{CASE REPORT}

The authors had a 38-year-old patient presented to the clinic with the chief complaint of a painful indurated ulcer measuring $2 \times 3 \mathrm{~cm}$ at the interscapular region, with pus discharge for 2 days. The patient had no history of diabetes, HIV infection, or prolonged corticosteroid therapy. The patient reported no trauma and was unsure about insect bite. The patient was admitted to wards and started on cefoperazone sulbactam and tramadol. Debridement of the ulcer was undertaken on Day 1 of admission. On post-operative Day 2, the entire wound was filled with pus and black discolouration. Clinical examination of the infected area revealed a large black necrotic patch measuring $6 \times 6 \mathrm{~cm}$ with a satellite lesion measuring $1.5 \times 2.5 \mathrm{~cm}$ at the interscapular region (Figure 1).

On the second hospital day, debridement was repeated, and the sample was sent for microbiology and histopathology work-up. Daily dressing of the wound was undertaken with betadine (povidone-iodine) and hydrogen peroxide, and the patient was continued on medications. On histopathology exam, the findings were thick, broad, non-septate fungal hyphae suggestive of mucormycosis (Box 1).

Systemic amphotericin B was started on the day mucormycosis was confirmed. Simultaneously, daily local dressings were undertaken for the wound with povidone-iodine, hydrogen peroxide, and normal saline, along with topical amphotericin B deoxycholate.

Extensive surgical debridement was done on the fourth hospital day, and the patient was continued on amphotericin $B$ and routine wound care (Figure 2). The patient developed fever and chills which improved with supportive care. The patient's wound showed improvement over next 3 weeks from pale granulation tissue to healthy granulation tissue (Figure 2). Splitskin grafting was performed using auto graft from the left thigh under general anaesthesia. The graft showed $100 \%$ uptake with a healthy wound (Figure 3).

The patient was continued on a single dose of amphotericin B at $1 \mathrm{mg} / \mathrm{kg}$ body weight as an infusion in $100 \mathrm{~mL}$ of $5 \%$ dextrose over 1-2 hours for a period of 45 days. The patient was doing well after 20 months since hospital discharge.

\section{DISCUSSION}

Mucormycosis is the common name given to several different diseases caused by fungi of the order Mucorales. Mucormycosis is an opportunistic fungal infection, usually occurring in patients who are immunocompromised but can infect healthy individuals as well. Predisposing factors for mucormycosis are uncontrolled diabetes, malignancies such as Iymphoma and leukaemia, renal failure, organ transplant, long-term corticosteroid use and immunosuppressive therapy, cirrhosis, burns, protein-energy malnutrition, and AIDS. The current patient was a young male with full immunocompetency and no comorbidities.

In patients who are immunocompetent, phagocytosis of the spores prevents a fulminant course of the infection. Macrophages and neutrophils play an important role in this process. However, in individuals who are immunocompromised, lack of production and disruption of function of these immune cells lead to a rapid, progressive course of disease.

Manifestation of cutaneous mucormycosis is variable and can present gradually or as a fulminant disease leading to dissemination. ${ }^{17}$

This case report illustrates that the diagnosis of cutaneous mucormycosis can be difficult because initial symptoms are often non-specific and can mimic a variety of infectious skin diseases. This underlines the utmost importance of early harvesting of soft-tissue probes from the lesion site, in cases of progressive signs of infection. ${ }^{18}$ Biopsy samples should be attained early, so treatment can be initiated. Pathological features include angioinvasion, which initiates thrombosis and infarction of the affected surrounding tissues, leading to necrosis. The presence of wide, twisted fungal hyphae within blood vessels, with necrosis of the tissues supplied by affected vessels, is diagnostic for mucormycosis. Microbiological studies can then delineate the species of fungi involved. ${ }^{19}$ 


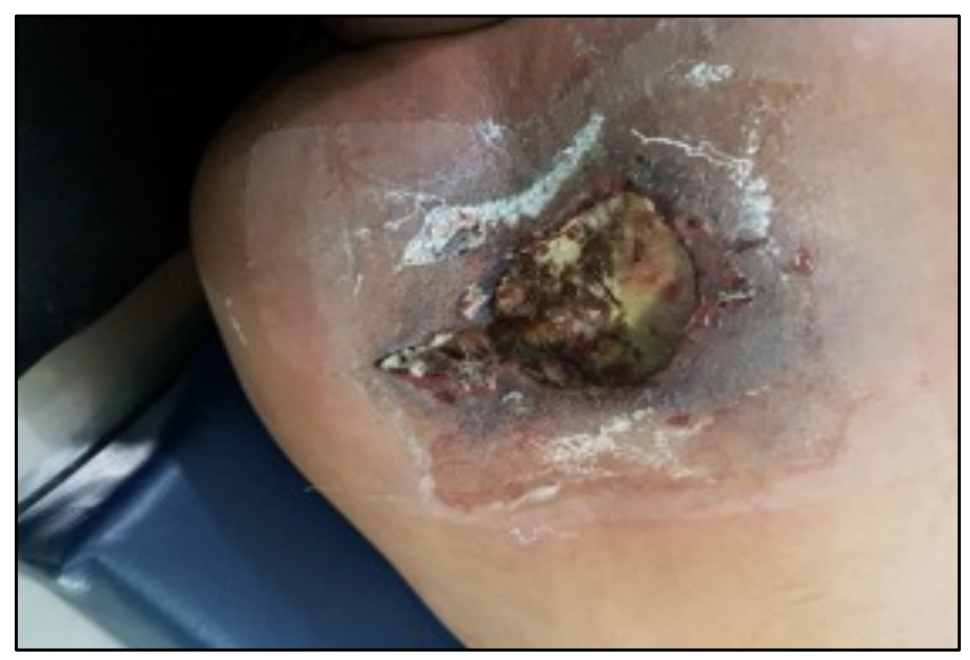

Figure 1: Clinical presentation of patient after first debridement.
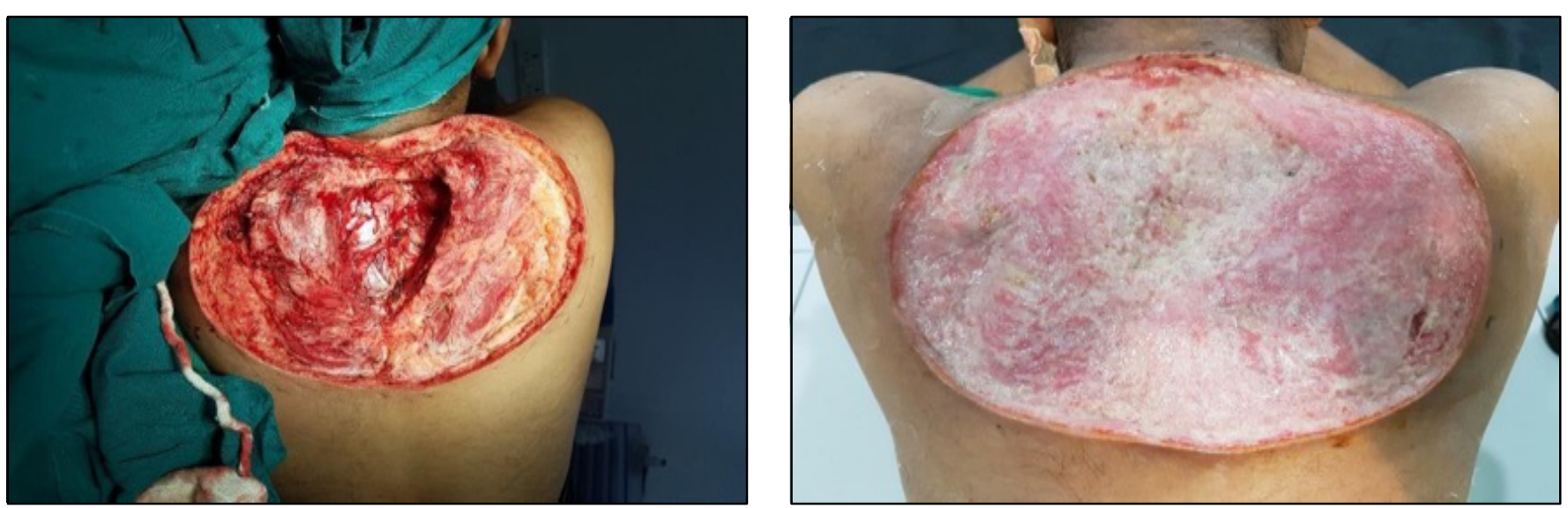

Figure 2: Final debridement (left) and healthy granulation tissue (right).

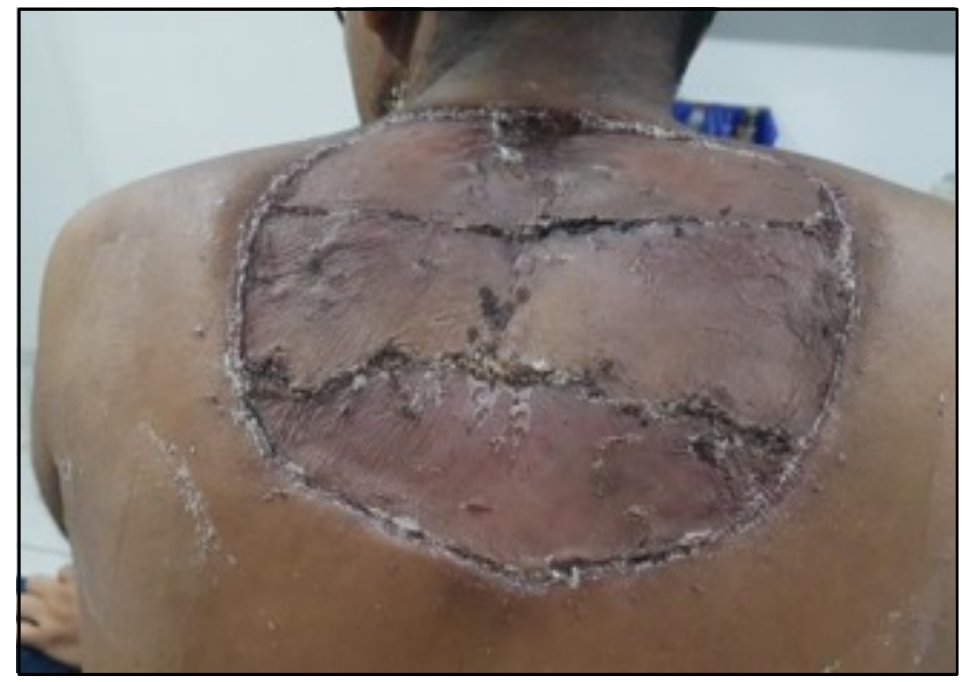

Figure 3: Post-skin grafting. 


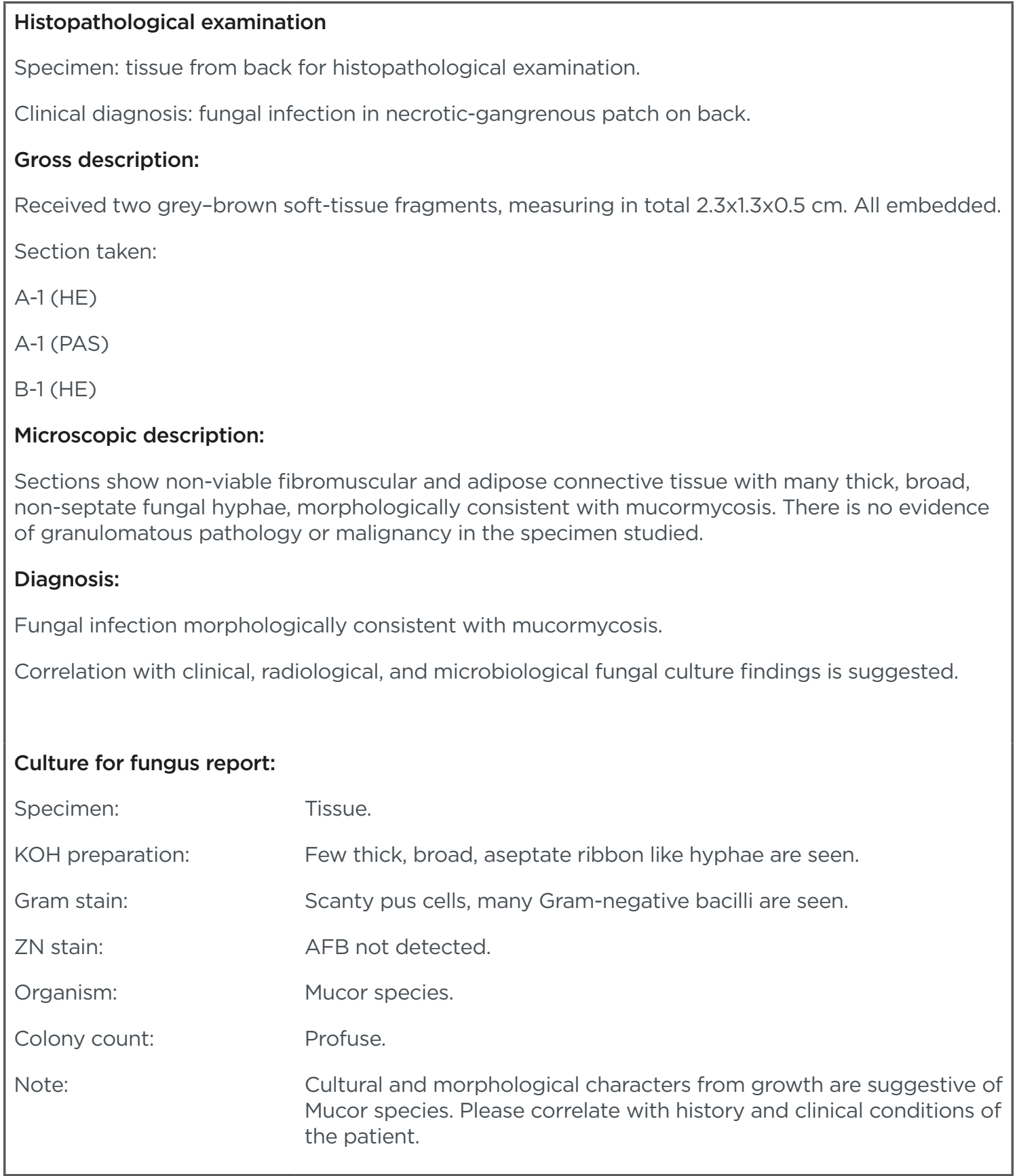

AFB: acid-fast bacillus; HE: haematoxylin and eosin; KOH: potassium hydroxide; PAS: periodic acid-Schiff; ZN: Ziehl-Neelsen.
A multi-modal approach in the management of cutaneous mucormycosis has been
manages the extensive necrosis occurring that demonstrated to improve overall survival. This involves reversing any risks and underlying contributing comorbidities, systemic antifungal treatment, and aggressive surgical debridement. Debridement optimises cure rates by preventing further dissemination to deeper organs and may not be prevented by killing the organism with antifungals. When combined with early, high-dose systemic antifungal therapy, studies have shown that mortality can be reduced to less than $10 \%$. Due to the resistance of Mucorales, the antifungal agent of choice is typically amphotericin B at high doses. However, because of its nephrotoxicity, 
renal function needs to be monitored. Novel regimens include using combination therapies (echinocandin or itraconazole) and adjunct treatments such as hyperbaric oxygen. ${ }^{20}$

\section{CONCLUSIONS}

The key to early detection and management of mucormycosis having a high index of suspicion for the disease. Ulcers with necrotic edges are not uncommon; however, its rapidly progressive nature should warrant suspicion of this disease. Identification of known risk factors, coupled with clinical findings and unresponsiveness to usual treatment, should prompt investigations. In this way, treatment can be initiated early to prevent a fulminant course of disease.

\section{References}

1. Hernández JL, Buckley CJ. Mucormycosis (2020), Treasure Island, Florida, USA: StatPearls Publishing.

2. Patel $\mathrm{A}$ et al. A multicentre observational study on the epidemiology, risk factors, management and outcomes of mucormycosis in India. Clin Microbiol Infect. 2020;26(7):944.

3. Castrejón-Pérez AD et al. Cutaneous mucormycosis. An Bras Dermatol. 2017;92(3):304-11.

4. Fehr $M$ et al. Multi-fungal sepsis and mucormycosis of the central nervous system in a patient treated with ibrutinib, a case report and review of the literature. Med Mycol Case Rep. 2020;27:14-6

5. Wang $Y$ et al. Cutaneous mucormycosis caused by Rhizopus microsporus in an immunocompetent patient: a case report and review of literature. Med. 2018;97(25):1-5.

6. Konigsberg MW et al. Topical treatment for cutaneous mucormycosis of the upper extremity. J Hand Surg. 2020;45(12):1189.e1-5.

7. Sharifpour A et al. Voriconazole associated mucormycosis in a patient with relapsed acute lymphoblastic leukemia and hematopoietic stem cell transplant failure: a case report. J Mycol Med. 2018;28(3):527-30.

8. Werthman-Ehrenreich A. Mucormycosis with orbital compartment syndrome in a patient with COVID-19. Am J Emerg Med. 2021;42:264.e5-8.

9. Elzein $\mathrm{F}$ et al. Mucormycosis: an 8-year experience of a tertiary care centre in Saudi Arabia. J Infect Public Health. 2020;13(11):1774-9.

10. Ibrahim A et al. Pathogenesis of mucormycosis. Clin Infect Dis. 2012;54(Suppl 1):16-22.

11. Bardwell J. Pulmonary mucormycosis in a heart transplant patient. Am J Med. 2020;133(9):524-5.

12. Jack AS et al. Brachial plexus mucormycosis secondary to perineurial spread: literature review and case report of a rare mode of infectious spread. Interdiscip Neurosurg. 2020;20:100687.

13. Seifert $\mathrm{S}$ et al. Pulmonary mucormycosis with extensive bronchial necrosis and bronchomediastinal fistula: a case report and review. Respir Med Case Rep. 2020;30:101082.

14. Oliveira Dos Santos RL et al. Rhinocerebral mucormycosis: diagnosis, treatment, and buccomaxillofacial rehabilitation Oral Surg Oral Med Oral Pathol Oral Radiol. 2020;130(3):116-7.

15. Uraguchi $\mathrm{K}$ et al. A case of rhinocerebral mucormycosis with brain abscess drained by endoscopic endonasal skull base surgery. Med Mycol Case Rep. 2020;30:22-5.

16. Ha NT et al. Brain lesion in a recreational drug user: isolated cerebral mucormycosis. IDCases. 2020;20:e00979.

17. Cornely $\mathrm{OA}$ et al. Global guideline for the diagnosis and management of mucormycosis: an initiative of the European Confederation of Medical Mycology in cooperation with the Mycoses Study Group Education and Research Consortium. Lancet Infect Dis. 2019;19(12):e405-21.

18. Binder $U$ et al. Mucormycosis-from the pathogens to the disease. Clin Microbiol Inft. 2014;20(Suppl 6):60-6

19. Nidhi $\mathrm{M}$ et al. Gastrointestinal mucormycosis in a two-year-old child: a clinical and radiological enigma. Med Mycol Case Rep. 2019;26:5-9.

20. Johnston J, Merchant A. Invasive primary gastric mucormycosisassociated gastric perforation in an immunocompetent host. Aust Crit Care. 2020;33(Suppl 1):S40. 